International Journal of Clinical Therapeutics and Diagnosis (IJCTD)

ISSN 2332-2926

\title{
An Unusual and Rare Cause of Meningitis in An Adult Renal Transplant Patient
}

Burton $\mathrm{H}^{1}$, Salter $\mathrm{T}^{1}$, Somalanka $\mathrm{S}^{1 *}$, Marsh $\mathrm{J}^{2}$

${ }^{1}$ Renal Registrar, Guys \& St Thomas NHS Foundation Trust, UK.

2 Joint Medical Director \& Consultant Nephrologist, Epsom \& St Helier University Hospitals NHS Trust, UK.

\begin{abstract}
Renal allograft recipients are on life long immunosuppressive medications. This predisposes them to serious infections such as meningitis. However, Escherichia Coli (EC) causing meningitis is uncommon, and extremely rare in adults. There is a dearth of reported cases in the literature. We report a first case of Escherichia Coli meningitis (ECM) in a renal transplant recipient who had undergone craniofacial surgery followed by radiotherapy for an ossifying fibroma associated with Hyperparathyroidism-Jaw Tumour Syndrome (HPT-JTS). Early recognition and choice of appropriate antibiotic therapy gave a successful outcome. Our case demonstrates the risks related to immunosuppression following renal transplantation, such as increased susceptibility to infections and decreased tumour immunosurveillance, which can contribute to potentially life threatening infections. Our case highlights the importance of clinical vigilance necessary in our patient cohort and discusses the pathophysiology of the virulence factors involved in the invasion of blood brain barrier (BBB) leading to ECM.
\end{abstract}

Keywords: Renal Transplantation; Immunosuppression; Meningitis; Tumour; Immuno Surveillance; Escherichia Coli.

\section{*Corresponding Author:}

Dr. Subash Somalanka B.Sc(Maths Hons) MBBS MRCP(UK, London), SCE(Nephrology),

Renal Registrar, Department of Renal Medicine, Renal Offices, $6^{\text {th }}$

Floor, Borough wing, Guys Hospital, Guys \& St Thomas NHS Foundation Trust, Great Maze Pond, London, SE1 9RT, UK.

E-mail: Nephr0nite@gmail.com

Received: March 26, 2015

Accepted: April 08, 2015

Published: April 10, 2015

Citation: Burton H, Salter T, Somalanka S, Marsh J (2015) An Unusual and Rare Cause of Meningitis In An Adult Renal Transplant Patient. Int J Clin Ther Diagn. 3(2) 81-84. doi: http://dx.doi.org/10.19070/23322926-1500016

Copyright: Somalanka $\mathbf{S}^{\circ} 2015$. This is an open-access article distributed under the terms of the Creative Commons Attribution License, which permits unrestricted use, distribution and reproduction in any medium, provided the original author and source are credited.

\section{Introduction}

Renal Replacement Therapy (RRT) in the form of kidney transplantation is the treatment of choice for end stage kidney disease [ESKD] with better outcomes in comparison to dialysis. However, transplantation gives an additional lifetime risk due to the burden of immunosuppression (IS). Severe life threatening opportunistic infections are common during the immediate and early post transplantation period due to heavy IS burden and decline during the long-term low/maintenance IS period. Meningitis can be a serious life threatening infection in immuno-suppressed patients. $\mathrm{ECM}$ is a commonly recognized infection in the neonates, but is an uncommon cause of bacterial meningitis in adults. A PubMed literature search revealed 2 case series: Copenhagen group [1977 - 97; $n=20]$ and a Taiwanese group [1986 - 2003; $n=15]$. The reported mortality rates varied between the groups with $40 \%$ in Copenhagen series and 100\% (untreated) versus 27\% (treated) in Taiwan series. We describe a case of a rare cause of meningitis in adult renal transplant patient which has not been described before. Our case report will add to the current wealth of available literature about ECM in adults and increase the awareness of being vigilant in patients on long-term IS.

\section{Presentation}

Our patient has HPT-JT characterized by the development of multiple parathyroid adenomas and fibro-osseous tumours of the maxilla and the mandible. He developed ESKD secondary to nephrocalcinosis in May 2010. Following a brief period on haemodialysis, he received a living donor kidney transplant in October 2010. He received an initial IS regimen, which consisted of induction therapy with basiliximab and 7 days of glucocorticoids, followed by maintenance therapy with tacrolimus and mycophenolate mofetil (MMF). A protocol biopsy at 3 months post-transplantation was performed in January 2011. This demonstrated Banff 1A/1B cellular rejection in the allograft, which was treated with 3-pulsed doses of methyl-prednisolone $(500 \mathrm{mg} /$ day) followed by oral prednisolone $(20 \mathrm{mg} /$ day). Serum creatinine remained stable between $120-135 \mu \mathrm{mol} / \mathrm{l}$. MMF was withdrawn by May 2011. He remained on maintenance IS with prednisolone $(5 \mathrm{mg} /$ day) and a dose of tacrolimus with target trough levels of $5-8 \mu \mathrm{g} / \mathrm{L}$.

His cranio-facial osseous fibroma became aggressive a year after 
transplantation for which he underwent craniofacial surgery in 2011 and again in June 2012. The surgery involved resection of the frontal bone, frontal cranial fossae, medial walls of the orbits, maxillary antra, nasal septum, turbinates and left nasal bone. He had reconstruction with fat to support his frontal lobes. There were no infective complications after his neurosurgical interventions on either occasion. Clinically, he was well with out any evidence of sepsis. A gadolinium-based magnetic resonance imaging in July 2012 prior to the commencement of radiotherapy did not reveal any inflammation or any infection. He required adjuvant radiotherapy due to the recurrence and aggressiveness of the tumour in August 2012. He became acutely unwell following a week after a session of radiotherapy with vomiting, fever, confusion and decreased responsiveness. He became febrile, hypotensive with tachycardia and tachypnoea. He showed signs of agitation and meningism. His glasgow coma score (GCS) was $10 / 15$ with bilateral up going plantar reflexes and absent focal neurological signs.

Blood tests revealed a white cell count of $29 \times 10^{9} / \mathrm{L}$ (neutrophil count of 26) with a C-reactive protein (CRP) of $8 \mathrm{mg} / \mathrm{L}$. Renal transplant function remained at its baseline. Blood tacrolimus concentration before this febrile episode was $6 \mu \mathrm{g} / \mathrm{L}$. Computed tomography scan of his head with contrast enhancement showed changes consistent with previous craniofacial surgery with a re- sidual tumour (Figure 1).

Intravenous hydrocortisone, ceftriaxone and acyclovir with oxygen therapy were administered immediately. Lumbar puncture demonstrated an opening pressure of $28 \mathrm{~cm} \mathrm{H_{2 }} \mathrm{O}$ with turbid cerebrospinal fluid (CSF), which contained 1,400 X 10\% $/ \mathrm{L}$ red blood cells, 44,700 X 10\% $/ \mathrm{L}$ white blood cells (100\% neutrophils) but revealed a negative gram stain. Following lumbar puncture, he received intravenous meropenem, teicoplanin and acyclovir as empirical therapy till CSF culture results were available. Six hours later, he deteriorated and required airway ventilatory support with adjuvant inotropic (noradrenaline) therapy in the intensive care unit.

CSF cultures grew EC sensitive to ceftriaxone, meropenem and gentamicin. Blood cultures from the day of admission also grew EC following 6 to $7 \mathrm{hrs}$ of incubation with the similar antimicrobial sensitivity profile. Serum CRP had rapidly risen to 235 . A course of IV meropenem was completed with vital organ support following which he made a full recovery from ECM.

\section{Outcome and Follow-Up}

Our patient remained well after successful treatment of this life threatening infection with no neurological sequelae relating to ECM. Serum creatinine remained stable at $116 \mu \mathrm{mol} / 1$.

Figure 1: This is a contrast-computed tomography of head showing resection of the frontal bone, the frontal cranial fossae, the medial walls of the orbits, the medial walls of the maxillary antra, nasal septum, turbinates and left nasal bone. Fat is grafted to the frontal cranial fossae to support the frontal lobes of the brain and a metal mesh covers the frontal bone defect. Lateral wall of the left maxillary antrum is expanded with disorganised trabeculation and shows bilateral recurrent disease of both orbits consistent with fibrous dysplasia of HPT-JTS.

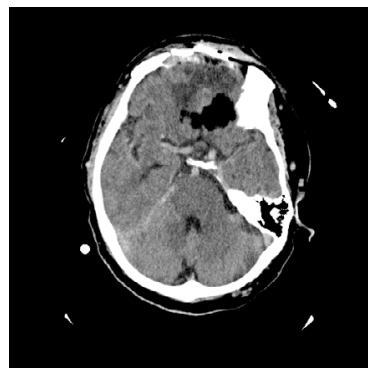

\section{Discussion}

This case is interesting in view of its complexity and shares the challenges one can face in the clinical management of solid organ transplant individuals. Renal transplantation remains the mainstay therapy for ESKD with improved outcomes and better quality of life. They are at an increased risk of infections due to their immunosuppressed state. Risk of infection is higher in the immediate post-transplant period when immunosuppression burden is higher. Infections, though can also occur later during the long-term low immunosuppression maintenance phase. There is often a greater risk of atypical infections in this group of patients. Meningitis is a serious life threatening infection and transplant recipients are at an increased risk of meningitis secondary to atypical organisms as compared to the general population. ECM is a well-known cause of neonatal meningitis but only a few cases of adult ECM have been reported and there has been no such report in a transplanted individual.

Here, we have described for the first time, a case of ECM in an adult renal transplant recipient, highlighting two important risk factors - increased susceptibility to infections and impaired tumour immunosurveillance. It is possible that these two factors have interacted simultaneously in our case. The growth of our patient's ossifying fibroma appears to have accelerated post-transplantation, necessitating more aggressive treatment (several craniofacial operations followed by radiotherapy) than would perhaps be expected for a benign tumour. It is possible that the radiotherapy may have caused shrinkage of the residual bony tumour with resultant exposure and breach in the bone/soft tissue interface thereby providing a route for EC to cross BBB and infect CSF. Radiotherapy therefore may have exposed our patient to the risk of infection, to which he had become more susceptible to by his immunosuppressive regime. This resulted in a life threatening ECM with out any co-infection with other organisms.

A PubMed search revealed 2 case series of meningitis that have been published: Copenhagen series had 20 consecutive cases from 1977 to 1997 [1] and a Taiwanese group who reported 15 cases from 1986 to 2003 [2]. These series estimate the percentage of all cases of adult bacterial meningitis caused by EC as $1.5 \%$ and 
$5 \%$ respectively. Another series from Taiwan reported $4.5 \%$ of their 233 cases to have ECM, of which $50 \%$ also had co-infection with Klebsiella Pneumonia [3]. There have been 8 case reports of ECM in adults which occurred after prostate biopsies [4-11]. Numerous other risk factors for ECM have been identified, including neurosurgical trauma/intervention $[3,12,13]$. Four cases were reported in post neurosurgical state with $50 \%$ mortality in one report [14], while in another reported review of 30 cases of gram negative bacterial meningitis, EC was the third most common pathogen [12]. While it is clinically intuitive that immunosuppression would have increased risk towards such an atypical infection leading to meningitis, ECM has only been reported in one case where there was co-infection with strongyloides stercoralis (SS) infection in a HIV-1 infected individual [15]. In another interesting case a fit adult, presented with bloody diarrhoea, fever, toxic mega colon and developed meningism and was found to have ECM. Disseminated SS infection leading to ECM was also reported [16-18]. While, EC is a facultative anaerobic gramnegative bacillus usually found in large intestine. A complicated SS infection might have exposed the gut blood barrier leading to meningitis. Our patient was well with out any gastrointestinal symptoms to explain the ECM. In another retrospective case review of 2,150 HIV-1 infected patients from 1985 to 1997, nine patients were found to have eleven episodes of purulent meningitis, of which only one episode was caused by EC [19]. This is interesting to note that even in a large immunosuppressed cohort, ECM incidence is rare. ECM has been reported in some cases with mycotic aortic aneurysms [20], Marfans syndrome [21, 22], caudal regression syndrome [23], pyelonephritis and urinary obstruction [24]. In a case series of 16 cirrhotic patients with meningitis, 9 were found to have CSF positive cultures for EC and Listeria monocytogenes of which 7 deaths were meningitis related with background of decompensated liver disease [25]. This series highlighted high mortality and the critically important factor of considering an appropriate choice of antibiotics for patient survival. The Taiwan series reported $100 \%$ mortality in the absence of appropriate antibiotics, whilst the use of appropriate antibiotic treatment reduced this to $27 \%$. The Copenhagen series reported a $40 \%$ mortality rate with the treatment.

Extra-intestinal pathogenic EC (ExPEC) has been implicated in severe infections such as osteomyelitis and meningitis. Particular genetic background of EC called phylogenetic group B2 has been associated with meningitis. In one particular study, multiple EC isolates from 4 adults with severe ExPEC infection including meningitis, isolated highly virulent phylogenetic type group B2 strain, which resembled typical isolates causing urinary infection and/or sepsis [26]. In neonates, virulence factor type K1 is mainly associated with ECM [27-29]. Moreover, antibiotic resistance has posed additional challenges, with the emergence of strains of E. coli that are not susceptible to third generation cephalosporins. ExPEC is known for developing multidrug resistance. Three clonal groups of ExPEC have been identified (O25b:H4B2-ST131, O15:H1-D-ST393 and CGA-D-ST69) which seem to cause majority of the ExPEC infections. A national survey in Spain collected 500 non-duplicate EC isolates in 2009 to determine virulence factors in these 3 clonal groups and found that clonal type O25b:H4-B2-ST131 accounted for 30\% of isolates with multi drug resistant ExPEC infections [30].

Our patient had non-extended spectrum beta lactamase EC infection that was sensitive to gentamicin, meropenem, ceftriaxone and ampicillin. We chose to avoid aminoglycosides to preserve his renal allograft function in the context of severe sepsis. Meropenem was chosen as a broad-spectrum beta-lactam bactericidal antimicrobial agent with a very successful outcome. We believe our report to be the first case of ECM in an adult renal transplant recipient with HPT-JTS. Our case also highlights the importance of increased vigilance for unusual and serious causes of infection in transplant recipients.

\section{Key Learning Points}

- Immunosuppression in renal transplant recipients predisposes to higher risk of unusual disseminated infections.

- Impaired tumour surveillance and increased susceptibility to infections are two important accepted risk factors in renal transplant recipients.

- E. Coli meningitis is common in neonates, rare in adults and can be life threatening in immunosuppressed individuals.

- Early detection and prompt consideration of appropriate antibiotic therapy offers better outcome.

\section{References}

[1]. Harder E, Møller K, Skinhøj P (1999) Enterobacteriaceae meningitis in adults: a review of 20 consecutive cases 1977-97. Scand J Infect Dis 31(3): 287-91.

[2]. Chang C-C, Lu C-H, Huang C-R, Chuang Y-C, Tsai N-W, et al. ( 2006) Culture-proven bacterial meningitis in elderly patients in southern Taiwan: clinical characteristics and prognostic factors. Acta Neurol Taiwan [Internet] 15(2):84-91.

[3]. Huang CR, Lu CH, Chang WN (2001) Adult Enterobacter meningitis: a high incidence of coinfection with other pathogens and frequent association with neurosurgical procedures. Infection 29(2):75-9.

[4]. Rodríguez-Patrón Rodríguez R, Navas Elorza E, Quereda Rodríguez-Navarro C, Mayayo Dehesa T (2003) Meningitis caused by multiresistant E. coli after an echo-directed transrectal biopsy. Actas Urol españolas 27(4):305-7.

[5]. Meisel F, Jacobi C, Kollmar R, Hug A, Schwaninger M, et al. (2003) Acute meningitis after transrectal prostate biopsy. Urologe A 42(12):1611-5.

[6]. Shen Z-J, Chen S-W, Wang H, Zhou X-L, Zhao J-P (2005) Life-threatening meningitis resulting from transrectal prostate biopsy. Asian J Androl 7(4): 453-5.

[7]. Alecsandru D, Gestoso I, Romero A, Martinez A, Garcia A, et al. (2006) E. coli multiresistant meningitis after transrectal prostate biopsy. Scientific World Journal 6:2323-6.

[8]. Samson D, Seguin T, Conil J-M, Georges B, Samii K (2007) Multiresistant Escherichia coli meningitis after transrectal prostate biopsy. Ann Fr Anesth Reanim 26(1):88-90.

[9]. Erdogan H, Ekinci MN, Hoscan MB, Erdogan A, Arslan H (2008) Acute bacterial meningitis after transrectal needle biopsy of the prostate: a case report. Prostate Cancer Prostatic Dis 11(2):207-8.

[10]. Nguyen B V, Cottrel M, Ralec B, Eyrieux S, Giacardi C, et al. (2009) A serious and unexpected infectious complication after transrectal prostate biopsy. Médecine Mal Infect 39(9):735-8.

[11]. Tufan ZK, Bulut C, Yazan T, Hatipoglu C, Erdinc S, Kinikli S, et al.( 2011) A life-threatening Escherichia coli meningitis after prostate biopsy. Urol J 8(1):69-71.

[12]. Lu CH, Chang WN, Chuang YC, Chang HW (1999) Gram-negative bacillary meningitis in adult post-neurosurgical patients. Surg Neurol 52(5): 438-43

[13]. Yang T-M, Lu C-H, Huang C-R, Tsai H-H, Tsai N-W, et al. (2005) Clinical characteristics of adult Escherichia coli meningitis. Jpn J Infect Dis 58(3): $168-70$.

[14]. Khan FY, Abukhattab M, Anand D (2013) Nosocomial Escherichia coli meningitis in adults: Report of four cases and literature review. J Neurosci Rural Pract 4(3):349-51.

[15]. Hovette P, Tuan JF, Camara P, Lejean Y, Lô N, et al. (2002) Pulmonary strongyloidiasis complicated by E. coli meningitis in a HIV-1 and HTLV-1 positive patient. 31(22):1021-3.

[16]. Smallman LA, Young JA, Shortland-Webb WR, Carey MP, et al. (1986) Strongyloides stercoralis hyperinfestation syndrome with Escherichia coli meningitis: report of two cases. J Clin Pathol 39(4):366-70.

[17]. Gomez JB, Maque Y, Moquillaza MA, Anicama WE (2013) E. coli Meningi- 
tis Presenting in a Patient with Disseminated Strongyloides stercoralis. Case Rep Infect Dis 2013:424362

[18]. Thompson AJ, Brown MM, Ridley A (1988) Escherichia coli meningitis and disseminated strongyloidiasis. J Neurol Neurosurg Psychiatry 51(12): 1596-7.

[19]. Almirante B, Saballs M, Ribera E, Pigrau C, et al. (1998) Favorable prognosis of purulent meningitis in patients infected with human immunodeficiency virus. Clin Infect Dis 27(1):176-80.

[20]. Weyrich P, Ettahar N, Legout L, Meybeck A, Leroy O, et al. (2012) First initial community-acquired meningitis due to extended-spectrum beta-lactamase producing Escherichia coli complicated with multiple aortic mycotic aneurysms. Ann Clin Microbiol Antimicrob 11:4.

[21]. Bigaré M, Lesaffre X, Delassus J-L, Bakir R, Bouldouyre M-A (2014) Relapse of Escherichia coli meningitidis due to sacral meningocele in Marfan syndrome, treated only with antibiotherapy. Presse Med 43(1):93-5.

[22]. Kangath RV, Midturi J (2013) An unusual case of E coli meningitis in a patient with Marfan's syndrome. BMJ Case Rep bcr2013008592.

[23]. Benson S, King M, Coulter C, Boyle RS (1993) Gram-negative meningitis and chronic constipation: an unusual presentation of caudal regression syndrome. Postgrad Med J 69(815):733-4.
[24]. Hasan S, Hall FC, Maidment G (1993) The Dambusters: a case of cerebrospinal and urinary obstruction. J R Soc Med 86(11):667.

[25]. Pauwels A, Pinès E, Abboura M, Chiche I, Lévy VG (1997) Bacterial meningitis in cirrhosis: review of 16 cases. J Hepatol 27(5):830-4.

[26]. Johnson JR, Gajewski A, Lesse AJ, Russo T (2003). Extraintestinal pathogenic Escherichia coli as a cause of invasive nonurinary infections. J Clin Microbiol 41(12):5798-802.

[27]. Huang SH, Stins MF, Kim KS (2000) Bacterial penetration across the bloodbrain barrier during the development of neonatal meningitis. Microbes Infect 2(10):1237-4.

[28]. Kim BY, Kang J, Kim KS (2005) Invasion processes of pathogenic Escherichia coli. Int J Med Microbiol 295(6-7):463-70.

[29]. Xie Y, Kim KJ, Kim KS (2004) Current concepts on Escherichia coli K1 translocation of the blood-brain barrier. FEMS Immunol Med Microbiol 42(3):271-9.

[30]. Blanco J, Mora A, Mamani R, López C, Blanco M, et al. (2011) National survey of Escherichia coli causing extraintestinal infections reveals the spread of drug-resistant clonal groups O25b:H4-B2-ST131, O15:H1-D-ST393 and CGA-D-ST69 with high virulence gene content in Spain. J Antimicrob Chemother 66(9):2011-21. 